\title{
LA MODALIDAD A DISTANCIA, ALTERNATIVA PARA LA CONSECUCIÓN DE LA MISIÓN DE LA UNIVERSIDAD POLITÉCNICA SALESIANA
}

\author{
(THE DISTANCE MODALITY, AN ALTERNATIVE TO ACHIEVE THE MISSION OF THE \\ POLYTECHNIC SALESIAN UNIVERSITY)
}

Edgar Loyola Illescas

Universidad Politécnica Salesiana (Ecuador)

\section{RESUMEN}

La Universidad Politécnica Salesiana del Ecuador, creada en 1994, en concordancia con las Políticas de la presencia salesiana en la Educación Superior en el mundo (Roma, 2003), desarrolla la gestión institucional con el propósito de ofrecer a la juventud una formación de calidad, que favorezca la inserción al mercado del trabajo y sobre todo incentivar y promover un compromiso social responsable que rebase las necesidades inmediatas del mercado y trascienda a un necesario cambio para mejorar la calidad de vida personal y social a la cual se pertenece.

La experiencia educativa de la UPS en sectores marginales de sociedad, la modalidad a distancia constituye una alternativa de alto impacto social, a través de la cual la Universidad concreta una de las características más sobresalientes de su misión institucional y que está referida a brindar una formación de calidad a jóvenes y estudiantes con limitadas posibilidades para realizar estudios a nivel superior.

Palabras clave: educación a distancia, evaluación, formación de calidad.

\section{ABSTRACT}

The Polytechnic Salesian University of Ecuador, created in 1994, according with the policies for the Salesian presence in Higher Education in the world (Rome, 2003), develops its institutional administration with the purpose to offer the youth a quality formation which helps the insertion to the 
labour market and mainly motivate and promote a responsible social commitment that surpasses the immediate market needs, and transcend to a necessary change to improve the quality of personal and social life to which we belong.

The educational experience of the Polytechnic Salesian University of Ecuador in marginal areas of society, the Distance Modality constitutes an alternative of high social impact, through which the University concretes one of the most outstanding characteristics of its institutional mission which is referred to offer a quality formation to young people and students with limited possibilities to achieve high level studies.

Key words: distance education, evaluation, quality formation.

\section{RECORRIDO HISTÓRICO INSTITUCIONAL}

La presencia universitaria salesiana más antigua en el mundo está presente desde 1934 en la India y luego desde 1940 con la Universidad Pontificia Salesiana en Roma.

En Ecuador los salesianos inician su labor pastoral-educativa a partir de 1888, formalizado en el Convenio firmado entre Don Bosco y el Representante del Gobierno de Turín-Italia, a través del cual se confía a los salesianos el Protectorado Católico de Artes y Oficios de Quito, para que impartan educación moral y científica a los hijos del pueblo para el desarrollo de la industria nacional, mediante la enseñanza sistemática de la artesanía.

Con la Ley de Creación publicada en el Registro Oficial 499 de la Presidencia de la República del Ecuador en 1994, la UPS inicia su vida jurídica con las facultades de Electromecánica, Ciencias Pecuarias y Agroindustriales y Ciencias Humanas y Sociales destacándose en ésta última el ámbito de las Ciencias de la Educación con las escuelas de Psicopedagogía, de Educación Bilingüe Intercultural y de Tecnología Educativa, y con los programas de Pregrado y Postgrado en Docencia Técnica y en Educación a Distancia.

La estructura organizativa vigente, responde directamente a la presencia universitaria con dimensión y cobertura nacional, cuenta con cuatro facultades en pregrado: Facultad de Ciencias Humanas y de la Educación, Facultad de Ingenierías, Facultad de Ciencias Agropecuarias y Ambientales y la Facultad de Ciencias 
Administrativas y Económicas; el postgrado se administra a través de la Unidad de Postgrados.

La población universitaria salesiana está constituida por aproximadamente 14000 estudiantes, 1000 personas entre docentes y administrativos, presentes en las ciudades de Cuenca, Quito y Guayaquil.

La misión institucional declara: La formación de honrados ciudadanos y buenos cristianos, con excelencia humana y académica. El desafío de nuestra propuesta educativa liberadora es formar actores sociales y políticos con visión crítica de la realidad, socialmente responsables, con voluntad transformadora y dirigida de manera preferencial a los pobres.

Amparados bajo esta misión que deviene de la Identidad y Políticas de las Instituciones Salesianas de Educación Superior (IUS), la UPS desarrolla su propuesta formativa, en varios sectores sociales urbanos y marginales del país.

De la totalidad de la población estudiantil el 10\% aproximadamente, desarrolla sus estudios bajo la modalidad a distancia, estudiantes que en su gran mayoría son parte de comunidades indígenas y campesinas que habitan en sectores geográficos de la cordillera andina y oriente ecuatoriano.

Las áreas que actualmente asumen la modalidad a distancia son: Sociales, Educativa, Agropecuaria, Educativa y Administrativas tanto en el nivel de pregrado como postgrado.

La realidad académica institucional actual en general, demanda una necesaria e ineludible aplicación de las tecnologías de la información y comunicación, que se constituyen en un complemento de la modalidad presencial.

La modalidad a distancia, constituye una efectiva y concreta alternativa que permite ser fieles a la teleología institucional, realidad que se concreta en las diferentes experiencias educativas, mismas que se constituyen en bases firmes de proyección institucional en beneficio de la sociedad a la que se pertenece.

\section{ENFOQUE INSTITUCIONAL DE LA EDUCACIÓN A DISTANCIA (EaD)}

La propuesta educativa salesiana asume el Sistema Preventivo, basado en la razón, religión y amor, metodología pedagógica que se traduce en el acompañamiento 
y la voluntad de compartir con los jóvenes su vida, atendiendo sus verdaderas exigencias y valores, clave que en el ámbito educativo define de manera concreta el rol del docente, evidenciada en una actitud de orientación, apoyo y constante guía, que aporte y coadyuve al proceso de aprendizaje partiendo desde la realidad concreta de cada estudiante facilitando la concreción de las nobles aspiraciones de una verdadera educación.

Una de las finalidades principales del proceso educativo, constituye sin duda la formación integral de la persona, que permita la realización dentro de un equilibrio y normalidad tal que sea capaz de satisfacer las necesidades personales y comunitarias de índole corporal o física, psíquica, y finalmente la de índole espiritual.

La filosofía, pedagogía, en general las ciencias sociales y las distintas bifurcaciones que derivan de ellas, generan y formulan varias teorías, corrientes, hipótesis, experiencias, modalidades, con la finalidad de explicar, aplicar e inferir de la mejor manera el complejo y amplio mundo de la educación y formación.

La teoría del constructivismo, explica el conocimiento humano y los aspectos que involucra, como un proceso personal, en la que el individuo se desarrolla en plenitud conforme asimila información e interactúa con su contexto o entorno, característica sustantiva que destaca también la importancia e incidencia del rol principal que asume el estudiante como actor principal del proceso educativo.

La realidad y experiencia educativa, por mucho tiempo se desarrolló y a pesar de muchos aún se desarrolla bajo la premisa de que el profesor, maestro, catedrático enseña y el alumno o estudiante aprende, es decir un énfasis mayor en todo sentido al rol del docente.

Jean Piaget, cuando aborda el desarrollo cognoscitivo, hace referencia al proceso de equilibrio que se da entre la asimilación y la acomodación mismo que se evidencia en tres fases: equilibrio entre el esquema interno del sujeto y la realidad externa o percibida; entre los esquemas internos del sujeto; y finalmente un equilibrio traducido en la jerarquía entre los diferentes esquemas, por lo que se sostiene que la motivación hacia el aprendizaje es del estudiante y no constituye objeto de manipulación por parte del profesor, evidenciándose que el proceso de aprendizaje se da al interno del sujeto que aprende y que lo demás constituye un contexto que debe ser ante todo de apoyo, orientación y acompañamiento, fortaleciendo y generando una sinergia de y entre los diferentes factores que son parte inherente en un proceso de formación. 
En la publicación de Freire (1968) versión en portugués, cuyo título es $L a$ Pedagogía del Oprimido, metafóricamente lanza un grito de rechazo a la educación bancaria, educación que se puede resumir en una frase sencilla pero con mucho contenido: el maestro todo sabe y por lo tanto enseña, mientras que el alumno no sabe nada y por lo tanto tiene que ser enseñado.

Freire, fundamenta su propuesta de educación liberadora en la capacidad innata de la persona, haciéndoles conscientes de su mundo, de sus capacidades y limitaciones, de sus propios problemas y posibilidades de solución, en suma hombres y mujeres dueños de su propio pensamiento y cosmovisión del mundo en el cual se desarrollan, en que el rol principal del profesor-tutor será el de propiciar, generar, fortalecer un ambiente facilitador y de orientación a nivel personal y de equipo.

Según Ormrod (2003), "el aprendizaje se forma construyendo nuestros propios conocimientos desde nuestras propias experiencias", realidad que implica que el esfuerzo más importante y significativo proviene del estudiante que es el principal actor del proceso de aprendizaje; mientras tanto que, el rol principal de profesor será el de facilitador; es decir el que anima y genera un ambiente propicio para que el estudiante descubra por si mismo la lógica del objeto de estudio, apoyándose en el aporte y colaboración, fruto de un trabajo cooperativo y de equipo en un determinado contexto social.

Los grandes hitos que ha marcado el desarrollo de la comunicación en el mundo, iniciando con la aparición de la imprenta, luego con la aparición de los medios de audio y video y finalmente con la telemática, sumado a la nueva organización del conocimiento traducido en la multi -yuxta- trans-disciplinariedad, han incidido de manera directa, en la sociedad y por ende en el sector educativo, en el que quizá de manera lenta obliga a un cambio de mentalidad y actitud de docentes y estudiantes, frente a los retos de una educación en que los roles de los mismos deben sintonizar y complementarse para así asegurar la tan ansiada calidad educativa.

Una de las tantas evidencias que podemos constatar de este cambio lento pero al fin cambio, es el hecho que la población docente universitaria se encuentra en una constante capacitación y actualización de sus destrezas cognoscitivas, movidos ya sea por el interés personal de hacerlo (plena conciencia) y otros por las exigencias propias de permanencia, vigencia y en ciertos casos por la exigencia de la institución a la que pertenecen; otro ejemplo es aquel que da cuenta de la tendencia creciente que plantea la validez temporal de la titulación universitaria, a fuerza de la necesidad y pertinencia laboral-empresarial como del acelerado y rápido avance del desarrolloconocimiento, el mismo que se duplica aproximadamente cada cinco años. 
La UPS, al ser una institución de inspiración cristiana, con carácter católico e índole salesiana, es de suma importancia otorgar también una mirada desde lo que plantea la Iglesia Latinoamericana sobre el tema educación, a través de los documentos generados en las Conferencias Episcopales de Medellín, educación liberadora, que convierte al educando en el sujeto de su propio desarrollo, promueve su sentido comunitario y evangelizador; y la de Puebla, que plantea una educación evangelizadora, que, en síntesis, aspira a convertir al educando en sujeto, no sólo de su propio desarrollo, sino también al servicio del desarrollo de la comunidad: educación al servicio (Bellini, 1990).

Con estas breves referencias, podemos inferir y constatar que en el proceso educativo, el rol protagónico del aprendizaje lo tiene y debe asumir el estudiante, y que los roles secundarios del profesor y la institución deben asumir una actitud complementaria válida para asumir la realidad estudiantil que como constante tiene la diversidad en la justa medida de la misma condición estudiantil, quien por derecho supremo aspira una educación de calidad.

La UPS orienta su propuesta educativa de modalidad a distancia en los distintos enfoques y directrices, enunciados brevemente en los párrafos anteriores, cuya opción institucional es la de ofrecer a la población marginada de la sociedad de manera particular al sector indígena y campesino en las distintas regiones del país, su mejor esfuerzo al fortalecimiento de la identidad cultural, construcción de la interculturalidad y consolidación de un orden social respetuoso, participativo, equitativo y solidario.

Entre los objetivos institucionales de la EaD, está el de formar docentes identificados con la realidad socioeconómica, política y cultural de las nacionalidades indígenas, que dominen conocimientos y técnicas en los campos educativo y productivo del proyecto histórico de los pueblos, especialmente indígena y campesino, sistematizando la sabiduría, ciencia y tecnología en función de la dignificación del hombre especialmente de las nacionalidades indígenas y campesinos.

Luego de hacer presencia universitaria por cerca de doce años en localidades lejanas y de difícil accesibilidad, la UPS se consolida en una alternativa válida de formación y profesionalización universitario, haciendo uso de la modalidad a distancia. 


\section{EXPERIENCIAS Y CONTRIBUCIÓN EN MODALIDAD A DISTANCIA}

\section{Pedagogía}

El sistema educativo nacional del país requería iniciar cuanto antes un proceso de mejoramiento que asegure una adecuada y pertinente educación de la niñez y juventud ecuatoriana y sobre todo de aquellos que se encuentran en zonas geográficas desatendidas por el gobierno central y local.

Uno de los varios aspectos a ser mejorado es el docente, realidad compleja ya que intervienen muchos aspectos entre ellos la ausencia de una planificación y un plan nacional a medio y largo alcance desde los organismos responsables a nivel nacional, la formación y capacidad pedagógica de parte de los docentes, la limitación de infraestructura, materiales, bibliografía, la estabilidad laboral y el justo reconocimiento a la labor docente, y otros más.

Frente a esta problemática uno de los frentes de trabajo para mejorar esta realidad, en la cual la universidad ha contribuido y lo sigue haciendo, es la capacitación y formación de todos quienes hacen docencia, especialmente en los niveles educativos parvulario, básico y medio, en diferentes instituciones educativas de índole privado como fiscal, evidentemente delegada una función y responsabilidad tan delicada e importante para el desarrollo de la persona, de la familia, en definitiva para la sociedad entera, a personas que no tienen la formación y capacitación para garantizar la calidad en su trabajo, se puede inferir que los resultados no son nada halagadores, antes por el contrario son preocupantes y de intervención urgente por parte de todas la instancias involucradas en el proceso.

El Ministerio de Educación y Cultura en 1995 expide el Acuerdo Ministerial No 1435, mediante el cual suscribe el Convenio de cooperación interinstitucional con la UPS, a través del cual se profesionalizarán en ciencias de la educación, aquellas personas que se encuentran en ejercicio de la docencia y no cuentan con formación pedagógica.

\section{En los sectores suburbanos y rurales}

La creciente demanda educativa y la limitada capacidad de respuesta a las mínimas demandas sociales de parte de las instancias responsables de la educación en el país, ha determinado un fenómeno que en los casos más críticos se lo identifica 
como mercantilización de la educación, y por otro la ineficiencia, incapacidad y burocracia del sector fiscal, realidades que de parte y parte afectan de manera directa a la calidad educativa, ambientes en los que se dan las más acuciantes y diversas problemáticas, tales como el funcionamiento irregular de los centros educativos, personal sin la debida preparación, infraestructura y recursos limitados, y en ciertos casos ausentes.

Los principales destinatarios de este programa, son personas que están laborando en diferentes instituciones educativas particulares y fiscales ubicadas generalmente en sectores periféricos y rurales de las grandes ciudades, o provincias que normalmente sufren la desatención de un sistema centralizado.

\section{En el Oriente ecuatoriano}

La UPS presente también desde 1995 en ésta región del país, cuyas características de la realidad educativa tiene similares condiciones descritas en los párrafos anteriores, con el agravante de que las pequeñas poblaciones se encuentran muy dispersas en amplias zonas y no disponen de vías de comunicación sino de senderos, comprometiendo seriamente la movilidad de la población y la respectiva accesibilidad. En casos urgentes pequeñas avionetas prestan servicio social.

Para el oportuno desarrollo del programa, la UPS mantiene convenios de cooperación con instituciones que se encuentran en las capitales o ciudades más pobladas del Oriente, tales como Macas con el Instituto Pedagógico José Félix Pintado, y Sucúa con el Servicio Educativo Radiofónico Bilingüe Intercultural Shuar (SERBISH).
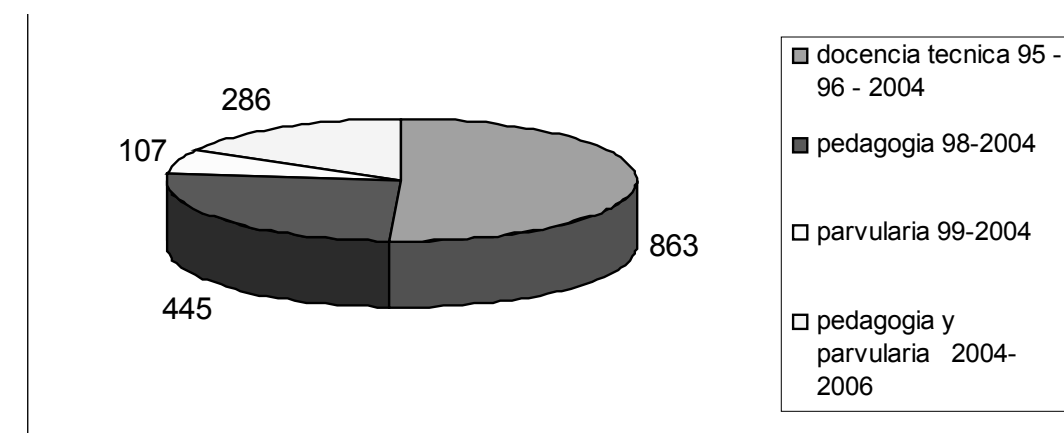

Gráfico 1- Relación entre el número de estudiantes y el área de especialización en la carrera de pedagogía (2006). 


\section{En la Cordillera Central de los Andes}

\section{Programa Académico Cotopaxi}

Esta experiencia universitaria se desarrolla en un sector geográfico ubicado en la parte central de la cordillera de los Andes, en cuyas estribaciones se encuentran las provincias mayoritariamente pobladas de ecuatorianos pertenecientes a las poblaciones indígenas bilingües (quichua - español), cuyos indicadores de bienestar social las ubican entre las provincias menos desatendidas por el gobierno nacional y local.

Entre los fines y objetivos de esta propuesta educativa está:

- Ofrecer, preferentemente a la población indígena y campesina de la región de la sierra central del país un servicio educativo de excelencia a nivel superior, orientado al fortalecimiento de la identidad cultural, construcción de la interculturalidad y consolidación de un orden social respetuoso, participativo, equitativo y solidario.

- Formar docentes identificados con la realidad socioeconómica, política y cultural de las nacionalidades indígenas, que dominen conocimientos y técnicas en los campos educativo y productivo del proyecto histórico de los pueblos, especialmente indígena y campesino.

- Sistematizar la sabiduría, la ciencia y la tecnología en función de la dignificación del hombre especialmente de las nacionalidades indígenas y campesinos. Formar, preferentemente, al personal docente que presta servicios en las jurisdicciones de Educación Intercultural Bilingüe, en las especialidades de docencia parvularia, docencia básica y docencia para el desarrollo comunitario.

- Realizar investigaciones de carácter social y tecnológico en función del desarrollo, especialmente del sector rural.

- Desarrollar un proceso de capacitación y actualización para dirigentes, comuneros, moradores de barrios populares, acerca de aspectos socioorganizativo, educación política, administración comunitaria y gerencial. 
La oferta académica considera: Docencia Parvularia Intercultural Bilingüe, Docencia Básica Intercultural Bilingüe y la Docencia y Desarrollo Comunitario, y los destinatarios del mismo son personas pertenecientes a la población rural, indígena de las provincias de Cotopaxi - Latacunga: 86 estudiantes, Imbabura - Otavalo: 169 estudiantes, Pichincha - Cayambe: 104 estudiantes, Bolívar - Simiatug: 119 estudiantes y Morona Santiago - Wasakentsa: 9 estudiantes, en un total de 487, de los cuales el $65 \%$ son mujeres y sus edades oscilan entre 18 y 68 .

\section{Antropología}

La Carrera de Antropología Aplicada es el resultado de la necesidad expresada por varios actores sociales vinculados con diversas instancias organizativas e institucionales, constituyéndose la modalidad a distancia una oportunidad de estudio para líderes que requerían de herramientas teóricas para profundizar su acción.

La propuesta académica, sin embargo de su condición de modalidad a distancia contempló espacios de carácter presencial en el que se desarrollaban grandes foros de reflexión teórico práctico, que permitían el intercambio de experiencias y ricos debates que articulaban las temáticas antropológicas con las necesidades de análisis local, nacional y regional.

En el auge de la oferta contó con estudiantes de algunos países de América Latina, tales como Argentina, Brasil, Paraguay, Bolivia, y Guatemala.

Entre los logros más destacados en este programa sin duda es la contribución a la sociedad con profesionales con mentalidad crítica, reconocidos en diversos espacios de trabajo teórico.

\section{Gestión para el Desarrollo Local Sostenible}

A partir del año de 1996, un grupo interdisciplinario recoge los principios del Desarrollo Local Sostenible para traducirla en una propuesta educativa, está siendo ejecutada en los últimos años logrando profesionalizar a actores/as locales que evitarán el agotamiento paulatino de la naturaleza, y al deterioro de los recursos naturales.

Este grupo interdisciplinario, liderado por Universidad Politécnica Salesiana (UPS) durante los años 1996 y 1997, con el aporte de varias organizaciones como: 
Fundación Interamericana (FIA), COMUNIDEC, ESQUEL, Programa Bosques, Árboles y Comunidades Rurales (FAO), Centro Bartolomé de las Casas, entre otros, se formula y diseña la propuesta académica orientada a la profesionalización con visión integradora del desarrollo.

La propuesta responde a una demanda de técnicos en desarrollo local, con conocimientos en: fortalecimiento institucional, políticas públicas, participación social, finanzas públicas, manejo ambiental, manejo de metodologías participativas, etc., y que se encuentran en instancias institucionales como: Municipalidades y Consejos Provinciales, Juntas Parroquiales, Organizaciones Sociales, Organizaciones de los Pueblos Indígenas y Negros, ONGs.

El objetivo central del programa es contribuir a la formación de profesionales del desarrollo local sostenible desde una perspectiva humanista intercultural e inter- y transdisciplinar, orientada a la construcción de una sociedad plural y equitativa.

Los datos de seguimiento de los últimos cuatro años de los estudiantes de este programa señalan que el $38 \%$ de estudiantes se pertenecen a los pueblos indios, el $6 \%$ de los pueblos negros, el $54 \%$ de los pueblos mestizos y finalmente el $2 \%$ de estudiantes extranjeros.

\section{Enfoque pedagógico}

El enfoque pedagógico que se aplica en el desarrollo educativo de la propuesta de gestión para el desarrollo local sostenible, considera la experiencia personal y colectiva como fundamento para la formulación de conceptos, misma que es descendida al campo de la aplicación, en la cual se recrea la vivencia, que a su vez como insumo enriquece la reflexión.

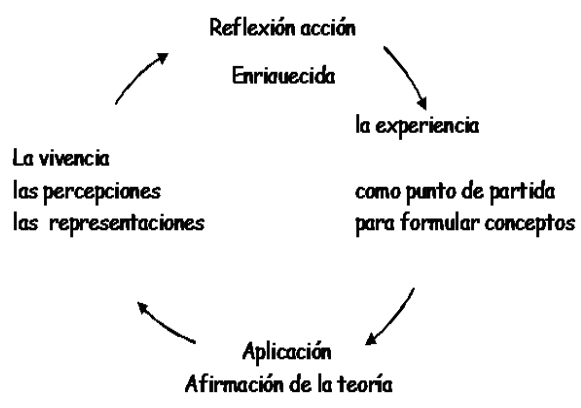


Algunos datos que ilustran rápidamente los resultados alcanzados, en una entrevista aplicada a 120 involucrados en el proceso:

- Reconocen que potencia sus capacidades de los líderes locales (119)

- Se sirven de herramientas metodológicas para democratizar la gestión (90)

- Reconocen que se forman como personas con un gran compromiso social y una responsabilidad por el cuidado de la tierra, (109)

- Mejoran sus capacidades para resolver problemas (115)

- Aplican lo que aprenden en los ejes de desarrollo, gestión, ambiental y metodológico (101)

- Reconocen que incorporan en la vida cotidiana y en su escenario de gestión la equidad de género (118)

\section{Agropecuaria}

Un aporte sustantivo desde la perspectiva institucional, es el desarrollo del programa de capacitación en agropecuaria, que considera titulaciones intermedias y de fin de carrera, generando la flexibilidad de cada estudiante desde su propia decisión y que pueda o no continuar sus estudios hasta el nivel de formación que considere óptimo y pertinente a su propia realidad.

Este programa tiene la característica de validar y mejorar el conocimiento empírico que tiene el estudiante y que proviene de su natural relación con las tareas de responsabilidad cotidiana. Es por ello indispensable que el estudiante esté vinculado a una unidad productiva.

Las actividades prácticas las realizan en los terrenos que en unos casos son propietarios o pertenecen a la cooperativa comunal de la cual es miembro, transformándose así dentro del argot académico, en laboratorios donde el estudiante complementa su formación tecnológica y experimental.

El desarrollo de la actividad académica entonces consta de un estudio a distancia, complementado con encuentros presenciales in situ para discutir y buscar alternativas de solución entre compañeros, conformando cooperativas de estudio integradas por estudiantes, según la ubicación geográfica; $y$, tutores que realizan el respectivo seguimiento y evolución de la Unidad Producción Agropecuaria (UPA).

La necesidad nace en 1999 de la comunidad indígena denominada Santo Domingo 2, misma que necesita de una población joven con educación superior en 
agropecuaria, sin la necesidad que los estudiantes tengan que desvincularse de las unidades productivas.

Para concretar y desarrollar la oferta en las condiciones señaladas, la universidad trabaja cooperativamente con organizaciones comunitarias indígenas y organismos internacionales.

En los gráficos siguientes se presentan algunos datos que informan sobre este programa académico, en los tres últimos períodos académicos.

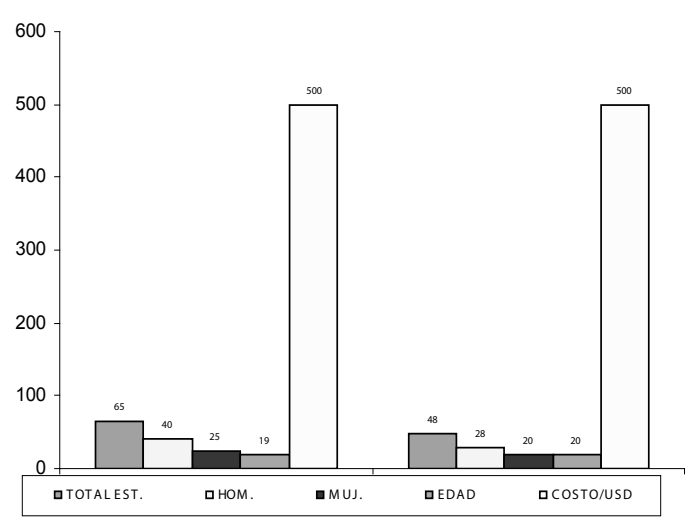

Gráfico 2 - Estudiantes: número, sexo, edad, costo

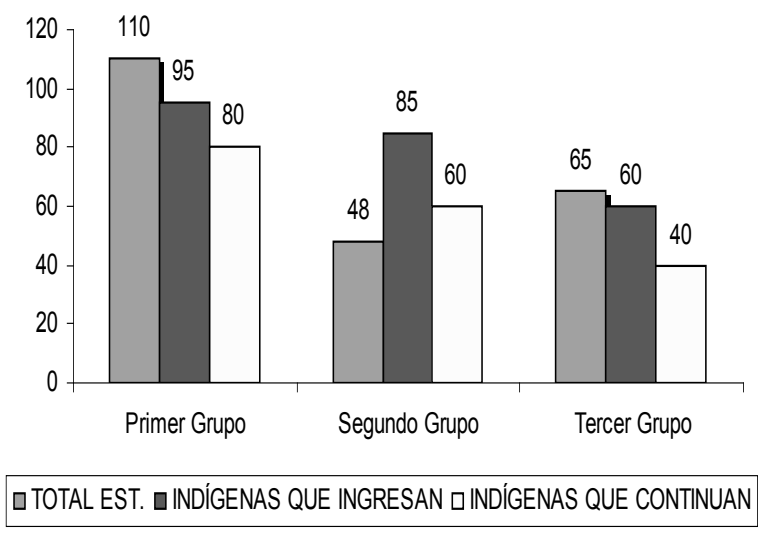

Gráfico 3 - Tendencia en las tres últimas promociones académicas 


\section{Curso virtual de las IUS para la Escuela América}

La organización denominada IUS (Instituciones Salesianas de Educación Superior) que la conforman más de 50 organizaciones salesianas repartidas en los distintos continentes, que suman sus mejores esfuerzos con la finalidad de cumplir y asegurar la presencia salesiana y superior bajo una identidad y políticas que amparen y orienten su labor pastoral y educativa. En este contexto en Roma (1998) mediante una asamblea se acuerda poner en marcha procesos con corresponsabilidad, generando sinergia, coordinación y continuidad.

Los puntos centrales de las IUS se refieren a la voluntad común, un objetivo común expresado en una visión compartida y finalmente un modo de proceder cooperativo, concretándose estas intenciones a través de los denominados Programa Común 1 (1998-2002), Programa Común 2 (2003-2007) y Programa Común 3 $(2008-\ldots)$.

Este curso responde a la necesidad de tener un denominador común de competencias y profesionalidad universitaria como verdaderos educadores en espíritu y estilo salesiano (identidad), una plataforma que permita proyectar y realizar juntos el proyecto institucional. El propósito central de esta formación totalmente en línea es dar significación integral a una realidad que dispersa a la pedagogía, las tecnologías de la educación y el estilo salesiano de formar.

Los beneficiarios de este curso son alrededor de 400 docentes de las instituciones salesianas educativas universitarias y no universitarias de Venezuela, Colombia y Ecuador.

Este programa académico se desarrolla en un entorno totalmente virtual, mismo que se denomina SOL (Salesiana Online) plataforma desarrollada y ajustada a los requerimientos institucionales y ejecutados sobre software libre, disponible desde la página Web institucional (www.ups.edu.ec).

\section{Testimonio estudiantil}

Finalmente, luego de hacer una breve reseña sobre los distintos programas académicos que desarrolla la UPS y sólo posibles a través de las ventajas y flexibilidad que otorga la modalidad a distancia, es importante compartir con los lectores la opinión de los estudiantes de la población indígena de Cayambe en la cual se desarrolla el programa académico en el área agropecuaria. 
Esta intervención es de un representante estudiantil, que cursa el último ciclo académico de la carrera de ingeniería agropecuaria, quien realizó esta presentación en el IV Encuentro de Planificación y Organización Universitaria, llevada a cabo en Cuenca julio 2006, bajo el tema la modalidad a distancia en la Universidad Politécnica Salesiana.

\section{Presencia de la UPS en la zona}

Debería iniciar señalando como experiencia personal y de muchos compañeros, que la vivimos hace siete u ocho años atrás, donde no existían ofertas de educación superior en el cantón, razón por la cual muchos de nosotros tuvimos que buscar oportunidades de estudiar fuera del cantón específicamente en Quito o Ibarra, y aun peor la gran mayoría de los jóvenes bachilleres de la zona optaban por no seguir estudios a nivel superior.

Desde este punto de vista el solo hecho de haber logrado la presencia de la Universidad Politécnica Salesiana en el Cantón ya fue un gran beneficio por que al fin los jóvenes y bachilleres en general de las comunidades aledañas al cantón íbamos a tener la oportunidad de acceder a una institución de estudios superiores sin la necesidad de salir fuera del cantón y lo mas importante, teníamos la oportunidad de combinar el estudio y el trabajo al mismo tiempo.

Ahora, la universidad estaba, la infraestructura a medida de las posibilidades estaba allí, pero la pregunta o preocupación que todos los estudiantes teníamos en un inicio era ¿cómo se va a encaminar y conseguir todas las propuestas que la universidad se las planteó para que nosotros como estudiantes las cumpliéramos a corto, mediano y largo plazo?

Hoy en día la interrogante que muchos de nosotros como estudiantes nos la hicimos en un gran porcentaje están resueltas, la universidad como tal ha tenido un gran impacto favorable en el desarrollo de las comunidades del canto Cayambe, Pedro Moncayo y otras zonas aledañas a los dos cantones, lo que nos ha permitido demostrarnos a nosotros mismos como estudiantes, a los integrantes de nuestras comunidades a las cuales pertenecemos que el prepararse profesionalmente no es sinónimo de buena posición económica, tampoco es sinónimo de gente blanca, el preparase, para nosotros es sinónimo de esfuerzo, constancia y dedicación.

El cómo la universidad ha logrado esto, no lo sé, pues muchos compañeros hemos sido testigos de los malabares, como halla decimos, que nuestro director 
de carrera con un grupo de amigos, como él en son de broma suele llamar a los docentes, han hecho para sacar adelante la propuesta de la universidad. Lo que sí les puedo comentar es que al momento los estudiantes de la Universidad Politécnica Salesiana Campus Cayambe, nos encontramos involucrados como entes partícipes con criterio en las diferentes actividades que signifiquen desarrollo para el cantón y por ende para sus comunidades.

Entonces la preparación no sólo ha sido en las aulas, hemos tenido la oportunidad de estar con la gente en las comunidades relacionadas con el campo agropecuario, tomando responsabilidades directas sobre el desarrollo económico de nuestras familias y comunidades, conociendo a profundidad la problemática del sector y proponiendo alternativas de solución.

Esto nos llena de satisfacción ya que la universidad nos ha dado la oportunidad de formarnos académicamente y a la par formar nuestro criterio sobre la realidad de la zona donde en un futuro nos vamos a desenvolver profesionalmente.

Me habían pedido un cometario sobre la presencia de la universidad en la zona y creo que les he manifestado lo que nosotros como estudiantes creemos, sentimos y pensamos de nuestra querida Universidad Politécnica Salesiana, cuya presencia la podemos desglosar en tres palabras: formación, participación y desarrollo.

\section{Calidad de la oferta académica}

Como estudiantes estamos concientes que nos ha costado en un pequeño porcentaje adaptarnos al sistema de educación semipresencial ya que muchos de nosotros venimos de colegios normales con un sistema de educación presencial en un $100 \%$, pero creo que en la actualidad hemos asimilado cual es la mecánica del sistema y hemos tomado hábitos de auto educación y de lectura, con lo cual los estudiantes tenemos la oportunidad de combinar el trabajo en las diferentes unidades productivas y estudiar al mismo tiempo.

Ventajosamente en este sentido como iniciativa de la universidad se han establecido mesas de discusión entre la dirección, catedráticos y estudiantes, las cuales han permitido tomar conjuntamente las mejores decisiones en lo que tiene que ver con la oferta académica.

La oferta académica que la carrera de ingeniería agropecuaria nos ofrece está a la altura de cualquier universidad del país, pero nosotros los estudiantes hemos 
asimilado que en este tipo de educación semipresencial no hay oferta académica que valga, si el estudiante no genera una capacidad de auto educación; de tal manera que él pueda combinar el trabajo de mejorar las unidades productivas a la que está vinculado y al mismo tiempo estudiar.

\section{Resultados}

Mas allá de lo anteriormente señalado como estudiantes creemos que la calidad de la oferta académica la podemos juzgar errada o acertadamente, en el desenvolvimiento que los estudiantes una vez formados profesionalmente tengamos en el lugar de trabajo.

En este sentido para nosotros las cosas se las está haciendo bien, por que hay compañeros que ya están trabajando conjuntamente con las comunidades y está generando desarrollo. Para nosotros lo más grato es tener en nuestras aulas al compañero Wilson Ushiña quien ganó el concurso BAYER dirigido a las universidades a nivel nacional.

\section{Los Docentes}

Como criterio personal creo que los estudiantes de la carrera somos privilegiados en este tema de los docentes porque hemos tenido la oportunidad de tener catedráticos de primer nivel, el sistema de estudio ha ayudado para que esto ocurra, por parte de la dirección se ha implementando talleres, seminarios, pasantías, etc., donde son invitados profesionales de prestigio conocedores de los diferentes temas relacionados con las cátedras.

\section{Materiales de estudio}

En este tipo de educación semi-presencial los estudiantes hemos asimilado que la herramienta mas importante para poder captar los conocimientos que nuestros tutores tratan de impartirnos es el material o la guía de estudio que al comienzo de cada periodo se nos entrega por cada cátedra.

Como material de estudio también tenemos un laboratorio que gracias a la universidad no ha sido sólo una herramienta de aprendizaje tutor - alumno, sino que se nos ha permitido realizar estudios a nivel macro buscando sanear problemáticas que nuestras comunidades tuvieren. 


\section{Recursos institucionales disponibles}

La universidad politécnica salesiana - campus Cayambe pese a tener una educación semi presencial se ha mostrado consolidada físicamente en el cantón, los estudiantes tenemos todas las comodidades que cualquier otra universidad puede brindar a sus estudiantes, existe un centro de computo con Internet disponibles 11 horas al día, se dispone de una muy bien dotada biblioteca, además se tiene un pequeño laboratorio que poco a poco lo han ido implementando.

Yo quisiera poner mucho énfasis en un recurso que quizá no sea institucional pero creo es igual de importante y me atrevería a decir que es una de las bases principales para la consolidación de la propuesta del Campus Cayambe, hablo de las comunidades aledañas al cantón, comunidades que han estado abiertas a compartir sus conocimientos ancestrales y a la vez aprender de nosotros.

\section{Atención institucional (Biblioteca-trámites-información-servicios varios)}

Creo que en este sentido no han habido problemas, ya que existe personal adecuado que nos ayuda rápida y eficazmente en cualquier necesidad eventual que podamos tener, como por ejemplo: solicitar libros, trámites de matrículas, información, etc.

\section{Costos}

Mas que el costo económico que significa estudiar en la Politécnica Salesiana por el mismo hecho de ser una institución privada, que para nosotros como estudiantes si es un valor considerable, quisiera resaltar las facilidades de pago que tenemos para cubrir el valor total de la matrícula, creo que la Politécnica Salesiana, allí marca la diferencia con el resto de las universidades particulares en el aspecto económico, ya que pese a ser una entidad particular la mecánica adoptada en el cobro de la matrícula permite que esté al alcance de estudiantes de cualquier nivel económico.

\section{CONCLUSIONES}

Desde luego uno de los indicadores de calidad de la gestión universitaria tiene que ver con el nivel de cercanía o distanciamiento que la institución educativa ha 
logrado con respecto a la declaración expresada en la misión institucional, y sentida ante todo por los sujetos del proceso educativo y por aquellos representantes de la compleja sociedad de la cual es parte la universidad.

Dejamos entonces en coherencia con lo mencionado en líneas anteriores, la opinión y criterio a los distinguidos lectores, para que juzguen y emitan un juicio de valor desde la luz de éste artículo que ha pretendido reflejar una realidad institucional en la que un porcentaje aproximado del 10\% de estudiantes realiza sus estudios bajo la modalidad a distancia.

La experiencia institucional a través de las diferentes ofertas académicas que funcionan bajo la modalidad a distancia, da cuenta que la misma facilita y vuelve factible llevar adelante alternativas de solución a los distintos problemas que devienen de los heterogéneos sectores que hacen la sociedad, problemas que se suscitan por la misma condición socio-económica de la población que proviene el estudiante y en sí mismo de la realidad inherente del estudiante, problemas que se generan de las limitaciones típicas de quien necesita y quiere capacitarse y sin embargo la estructura social lo impide.

La UPS, en el afán de contribuir en las áreas y aspectos que le corresponde y que tiene capacidad e ingerencia, desde la luz de su misión institucional, evidencia y constata de manera explícita y contundente el aporte de los que significa la modalidad a distancia, claro está que se tiene muchas limitaciones de índole general como lo constituye el ámbito educativo mismo y de las especificidades provenientes en el tema que se desarrolla la modalidad misma.

De ineludible responsabilidad es la capacitación y formación de los docentes y estudiantes en el nuevo rol que exige la educación actual, en la que cada actor debe estar mentalmente formado y dispuesto a trabajar de manera conjunta y complementaria en el proceso educativo, asumiendo las respectivas responsabilidades que demanda una formación y educación de calidad.

Otro aspecto y que no quiero dejar escapar, es el referido a la exclusividad o no de las ventajas y riesgos que nos trae las tecnologías de la información y comunicación, que por tendencia se suele asociarse con mucha facilidad a la educación no presencial, más considero que las mismas han incidido en toda la dimensión de la vida humana y por ello en todo lo que representa la educación, dejando la importancia que la tiene, desde luego en el sitio que corresponde a las disquisiciones de qué modalidad es mejor o peor, cuando sabemos que nos dirigimos a una educación que aprovecha 
lo mejor que tiene a su alcance el ser humano, para así concretar la tan ansiada y proclamada formación integral.

La calidad que ofrece una institución educativa está en directa proporción a la calidad de las personas que la integran, es por ello que el capital intangible de toda organización debe contar con la importancia concretada en el interés institucional de invertir en su mejoramiento.

\section{REFERENCIAS BIBLIOGRÁFICAS}

Bellini, L. (1990). Propuestas para una escuela popular desde los documentos de Medellín y Puebla. Roma: Ediciones Universidad Pontificia Salesiana.

Braido, P. (1994). Juan Bosco, el arte de educar. Escritos y testimonios. Madrid: Ediciones CCS.

Dicasterio para la Pastoral Juvenil (2000). La Pastoral Juvenil Salesiana, cuadro de referencia. Venezuela: Fundación Editorial Salesiana.

Dirección General Congregación Salesiana IUS (2003). Identidad de la Instituciones Salesianas de Educación Superior. Roma.

Dirección General Congregación Salesiana IUS (2003). Políticas para la presencia Salesiana en la Educación Superior. Roma.

Fernández, R. (2003). Competencias profesionales de los docentes en el siglo $X X I$. Profesor de nuevas tecnologías aplicadas a la educación. Cuenca: Ediciones Universidad de Castilla-La Mancha.

García, L. (1998). El material impreso en la enseñanza a distancia. Madrid: UNED.

García, L. (2001). La Educación a Distancia. De la teoría a la práctica. Barcelona: Ariel.

Perelló, J. (1996). Apuntes para una pedagogía no directiva. Quito: Ediciones UPS.

Varios (2006). IV Encuentro de Planificación y Organización Universitaria. Quito: Ediciones UPS.

Varios (2005). Aprendizaje cooperativo al estilo salesiano. [en línea] Disponible en: www.ucb.edu.br [consulta 2006, 18 de diciembre].

Varios (2007). Aulas Virtuales. [en línea] Disponible en www.ups.edu.ec [consulta 2006, 19 de diciembre] 


\section{PERFIL ACADÉMICO Y PROFESIONAL DEL AUTOR}

Edgar Loyola Illescas, profesor titular de la UPS-Ecuador, Vicerrector Nacional en funciones. Miembro de la comisión de creación de la UPS. Estudios de postgrado en evaluación de la calidad de la educación superior, educación a distancia, investigación socioeducativa.

E-mail: vicerrector@ups.edu.ec

DIRECCIÓN DEL AUTOR

Edgar Loyola Illescas

Universidad Politécnica Salesiana

Calle Turuhuaico 3 - 69 y Calle Vieja

Cuenca, Ecuador

Fecha de recepción del artículo: 23/12/06

Fecha de aceptación del artículo: 21/02/07 